Aim of the study: Flow cytometry has an important role in diagnosis and classification of B-cell lymphoproliferative disorders (BCLPDs). However, in distinguishing chronic lymphocytic leukemia (CLL) from small lymphocytic lymphoma (SLL) only clinical criteria are available so far. Aim of the study was to determine differences in the expression of common B cell markers (CD22, CD79b and CD20) on the malignant lymphocytes in the peripheral blood samples of CLL and SLL patients. Material and methods: Peripheral blood samples of $56 \mathrm{CLL}$ and $11 \mathrm{SLL}$ patients were analyzed by 5-color flow cytometry on the CD45/CD19/CD5 gate for CD22, CD79b and CD20.

Results: In the samples collected from the CLL patients, CD22 expression was detected in only $20 \%$ of patients in the low pattern, while in SLL patients the expression was medium and present in $90.9 \%$ of patients $(p<0.0001)$. For CD79b expression, statistical significance is reached both in the expression pattern, which was low/medium for CLL and high for SLL, and expression level $(p=0.006)$. The expression of CD20 was counted as the CD20/CD19 ratio. The average ratio was 0.512 in the CLL patients vs. 0.931 in the SLL patients $(p=0.0001)$.

Conclusions: The pattern of expression and expression level of CD22, $\mathrm{CD} 79 \mathrm{~b}$ and CD20 in peripheral blood could be used for distinguishing SLL from CLL patients.

Key words: CD20, CD79b, CD22, CLL, SLL.

Contemp Oncol (Pozn) 2014; 18 (1): 29-33 DOI: $10.5114 /$ wo.2013.38570

\section{Possible role of CD22, CD79b and CD20 expression in distinguishing small lymphocytic lymphoma from chronic lymphocytic leukemia}

Danijela Jovanovic ${ }^{1}$, Predrag Djurdjevic ${ }^{1}$, Nebojsa Andjelkovic ${ }^{1}$, Ljubica Zivic ${ }^{2}$

${ }^{1}$ Hematology Clinic, Clinical Centre Kragujevac, Serbia

${ }^{2}$ Medical Faculty Kragujevac, Serbia

\section{Introduction}

Diagnosis and classification of B-cell lymphoproliferative disorders (BCLPDs) has been a tempting goal of modern hematology. Since no antigen has been found to be specific in certain BCLPDs, a panel of antibodies detected by flow cytometry is used to help classify these disorders considering not only expression, but its level as well [1]. The intensity of expression of certain antigens was also suggested as a valuable discriminator of CLL from other BCLPDs, especially when considering CD20, CD79b and surface immunoglobulin light chain (slg) [2]. Thus a CLL score (Matutes score) was developed in 1994, revised in 1997, scoring 1 point for each of weak CD79b, positive CD23, positive CD5, negative FMC7 and weak slg, and suggesting CLL as a diagnosis in each case scoring 4 or more points [3]. However, in the era of cell phenotype based classification of the lymphoproliferative disorders three entities are believed to have the same immunophenotype pattern: monoclonal B cell lymphocytosis, chronic lymphocytic leukemia and small lymphocytic lymphoma [4]. Suggested criteria for distinguishing these disorders are merely clinical. The World Health Organization classification of hematopoietic disorders describes CLL as leukemic, lymphocytic lymphoma, being only distinguishable from small lymphocytic lymphoma (SLL) by its leukemic appearance. Additional criteria state that CLL or SLL might be suspected in otherwise healthy adults who have an absolute increase in the clonal B lymphocytes but who have less than $5 \times 10^{9} /$ I B lymphocytes in the blood. However, in the absence of lymphadenopathy or organomegaly (as defined by physical examination and CT scans), cytopenias, or disease-related symptoms, the presence of fewer than $5 \times 10^{9} / \mathrm{I} B$ lymphocytes in the blood is defined as monoclonal B-lymphocytosis [1, 5].

Research in this field is mostly oriented at distinguishing monoclonal B-lymphocytosis as a benign condition from CLL and SLL, and so far has proved significant differences in lymphoid tissue homing receptors [5]. Other research considering common B cell markers (CD19, CD20, CD22 and CD79b) determined the differences between levels and patterns of expression of these markers in distinguishing normal vs. malignant B cells and CLL from other B cell lymphoproliferative disorders $[2,6,7]$. In our study we tested levels and expression patterns of common B cell markers CD20, CD22 and $\mathrm{CD79b}$ in order to determine differences of these parameters in the peripheral blood samples of clinically non-distinguishable cases of CLL and SLL.

\section{Material and methods}

Patient samples

The study includes peripheral blood samples from 67 patients (median age 66 years, range $45-81$, male : female ratio $2.04: 1$ ) referred for investiga- 
tion of lymphoproliferative disorder in the period of 20092011 at the Centre for Hematology, Clinical Centre Kragujevac, Serbia. All patients were tested for complete blood count with leukocyte differential, serum biochemistry, ultrasound of peripheral lymph nodes enlargement and liver and spleen size, as well as immunohistochemical analysis of bone marrow and lymph node biopsies. Patients who revealed lymphocytosis (more than $5 \times 10^{9} /$ l lymphocytes) had their peripheral blood tested on a flow cytometer in a panel for lymphoproliferative disorders. Diagnosis was made according to the IWCLL criteria [1]. After diagnosis establishment patients were divided into two groups. The first group consisted of 56 patients who after examination met clinical, biochemical and immunophenotypic criteria for the diagnosis of chronic lymphocytic leukemia, with absolute lymphocytosis in peripheral blood. The second group consisted of 11 patients who on presentation met biochemical and immunophenotypic criteria as in the first group, but clinical criteria defined diagnosis of small lymphocytic lymphoma. The second group of patients, after meeting the criteria for the watch and wait therapy option, were followed to the point when they developed lymphocytosis in peripheral blood in a count of more than $5 \times 10^{9} /$. Patients were followed monthly, and average time for lymphocytosis development was 12.8 months (range 3-21). Their peripheral blood samples were then tested by flow cytometry. In the first group of patients median lymphocyte count was $32.5 \times 10^{9} /$, range $18.3-223.5$, and in the second group it was $11.3 \times 10^{9} /$, range $7.6-47.4$.

\section{Flow cytometry}

Peripheral blood samples of patients in each group were analyzed using 5-color flow cytometry. In the first group a peripheral blood sample was taken at presentation, while in the second group it was analyzed after patients developed lymphocytosis. The analyzed sample was prepared using $100 \mu$ l of whole blood, incubated for $15 \mathrm{~min}$ at room temperature with $10 \mu \mathrm{l}$ of each monoclonal antibody. After incubation, erythrocyte lysis was performed with ImmunoPrep Beckman Coulter reagents (Cat. No. 7546999) on the Beckman Coulter TQPrep, and acquired immediately using an FC500 Beckman Coulter cytometer. Samples were analyzed in 5 tubes, presented in Table 1.

Antibody sources were as follows: CD45 FITC Beckman Coulter (Cat. No. A07782), CD3ECD Beckman Coulter (Cat. No. A07748), CD 20 e-CY5 Beckman Coulter (Cat. No. A07773), CD19 PE-CY7 Beckman Coulter (Cat. No.), CD21 FITC Beckman Coulter (Cat. No. IM0473U), CD5 PE (T1-RD1) Beckman Coulter (Cat. No. 6603020), CD23 ECD Beckman
Coulter (Cat. No. IM3609U), CD22 PE-CY5 Beckman Coulter (Cat. No. IM3704), FMC7 FITC Beckman Coulter (Cat. No. A07791), CD38PE Beckman Coulter (Cat. No. A07779), CD5pc5 Beckman Coulter (Cat. No. IM2637U), CD79b Beckman Coulter (Cat. No. IM1612), CD45ecd Beckman Coulter (IM2710U), kappa FITC Beckman Coulter (Cat. No. 6604287), lambda PE Beckman Coulter (Cat. No. 6604289).

The results were presented in the form of percentage of CD22, CD79b and CD20 positive cells in the group of CD19/ CD45/CD5 positive cells. For each antibody median fluorescence intensity was also measured. If more than $50 \%$ of cells expressed a certain marker it was stated as positive. According to the instrument settings, MFI values $0.5-1$ were stated as low, 1-3 as medium, and over 3 as high.

\section{Statistical analysis}

Differences in percentage of positive cells as well as median fluorescence intensity were assessed using Student's $t$-test in the statistical program SPSS 19.0. All p-values are two sided.

\section{Results}

Diagnosis of SLL/CLL was assessed in each patient using immunophenotypic CLL score (Matutes score), where all of the patients scored 4 or 5 . In each group there was no difference noted for either expression or median fluorescence intensity for markers CD5, CD23, CD19, FMC7, CD38, and CD45.

Results referring to common B cell markers CD 22 and CD79b are shown in Fig. 1.

Expression of CD22 in the CLL group of patients was present in $14.06 \pm 15.71 \%$, while in the SLL group it was present in $48.4 \pm 22.1 \%, p<0.000001$. Expression of CD79b in the CLL group of patients was present in $52.95 \pm 18.95 \%$ and in the SLL group in $68.99 \pm 18.75 \%, p<0.01$. Expression of CD 20 was present in $40.96 \pm 23.37 \%$ for the CLL group, and $67.31 \pm 15.47 \%$ for the SLL group, $p<0.001$. Especially interesting is the index of expression of these markers in comparison to CD19, where the CD20/CD19 index for the CLL group is $0.512 \pm 0.346$ and for the SLL group 0.913 $\pm 0.155 \%, p<0.0001$, illustrated in Fig. 2 .

The MFI values suggested low/negative CD22 (0.447482 $\pm 0.173317)$, low/medium CD20 (1.074964 \pm 0.66384$)$ and low/medium CD79b (1.7050179 \pm 0.8553711$)$ in CLL patients, while in SLL patients the expression pattern was high for CD20 (5.486364 \pm 3.764907$)$ and CD79b (3.6090909 $\pm 2.0507972)$ and medium for CD22 (1.175273 \pm 0.484996$)$, $p<0.001$ (Fig. 3). The MFI values for CD5, CD23, CD38, CD19 show no statistically significant difference.

Table 1. Markers used for investigation of cell surface phenotype in CLL and SLL

\begin{tabular}{|c|c|c|c|c|c|}
\hline Tube number & FITC & PE & $\mathrm{ECD}$ & $\mathrm{PE}-\mathrm{CY} 5$ & $\mathrm{PE}-\mathrm{CY} 7$ \\
\hline 1 & CD45 & CD5 & CD3 & CD20 & CD19 \\
\hline 2 & CD45 & CD5 & CD23 & CD22 & CD19 \\
\hline 3 & Kappa & Lambda & CD45 & CD20 & CD19 \\
\hline 4 & CD45 & CD79b (CB3-1) & C & CD5 & CD19 \\
\hline 5 & FMC7 & CD38 & CD45 & CD5 & CD19 \\
\hline
\end{tabular}



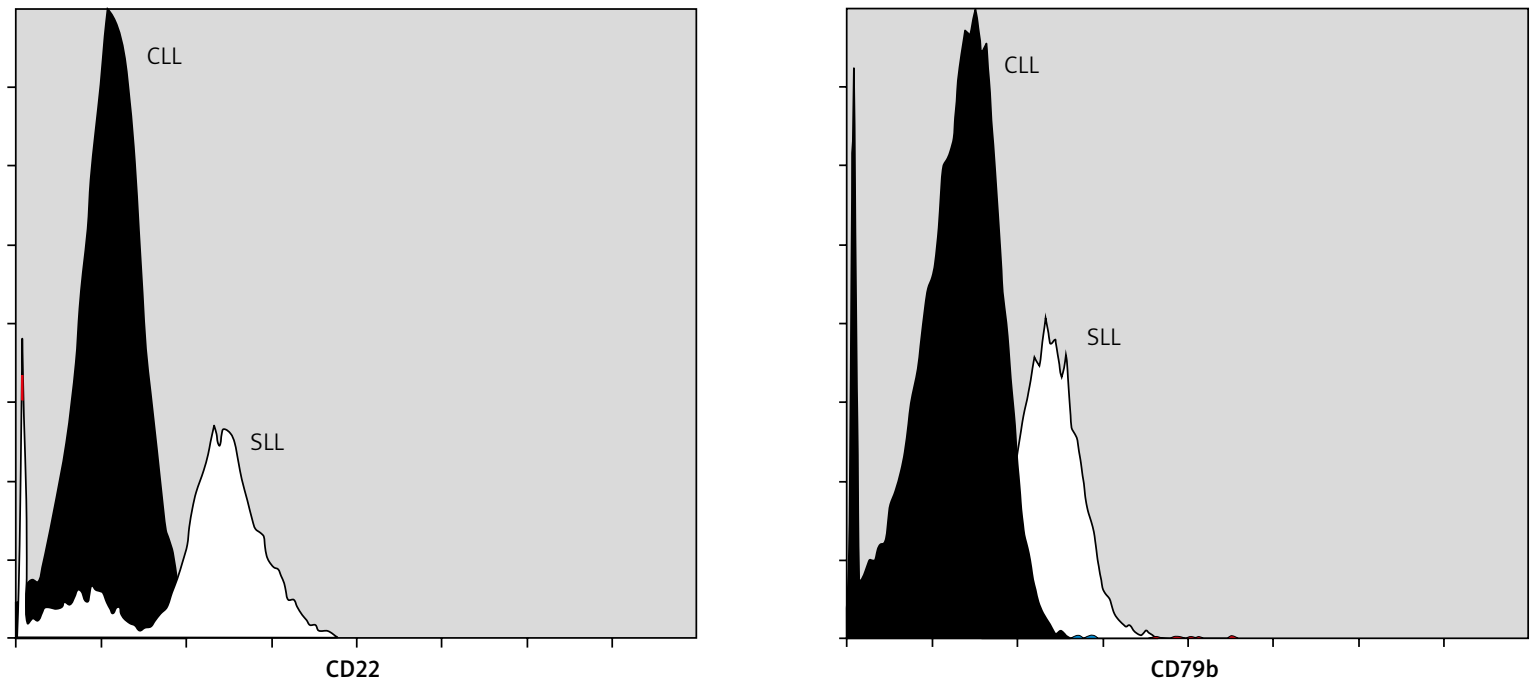

Fig. 1. Overlay histograms for CD22 and CD79b. Black histogram refers to CLL, white refers to SLL
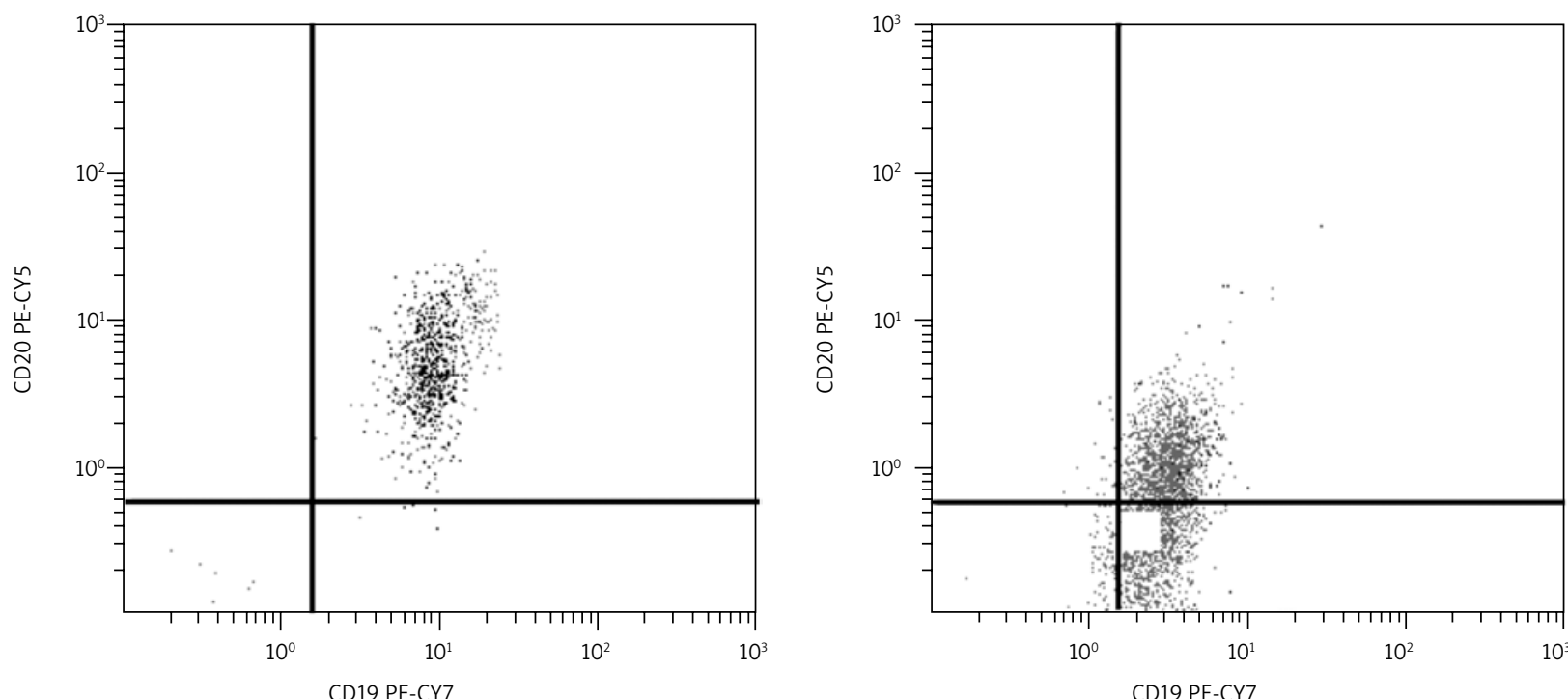

Fig. 2. CD19/CD20 expression in leukemic cells (SLL cells in the left plot and CLL cells in the right plot)

\section{Discussion}

The three molecules investigated here have a functional role in B cells. CD79b is a member of the B-cell receptor complex and forms a heterodimer with CD79a that is noncovalently associated with immunoglobulin [3, 8-10]. It is required for the transport of Ig to the membrane, for assembly and expression of B-cell antigen receptor signal transduction and the process of apoptosis. Its absence has a central role in the pathophysiology of chronic lymphocytic leukemia [11]. CD20 is a transmembrane phosphoprotein that functions as a calcium channel, and it has been shown to play an important role in B cell activation and differentiation. It appears later than other B cell markers during normal B lymphocyte development and its membrane density progressively increases during differentiation [7, 12, 13]. Biologically, it is an amplifier of calcium signals that are transduced through the BCR during antigen recognition by immature and mature B cells [14]. CD22 is a B-cell restricted sialoglycoprotein present in the

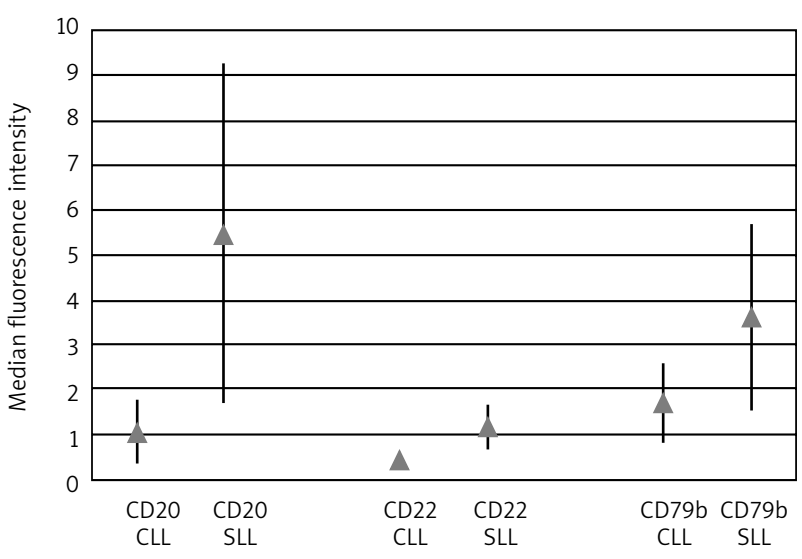

Fig. 3. Median fluorescence intensity of CD20, CD22 and CD79b in $\mathrm{CLL}$ and SLL samples (average value $\pm \mathrm{SD}$ )

cytoplasm of virtually all B-lineage cells but expressed on the B-cell surface only at mature stages of differentiation [15]. CD22 has two different functions on B-cells. It is well 
known as a negative regulatory molecule of the B-cell antigen receptor (BCR) signal leading to inhibition of B-cell activation. Moreover, CD22 is also considered as an adhesion receptor for the homing of re-circulating IgD positive B-cells in the bone marrow and lymph nodes via the expression of CD22 ligand on bone marrow and lymph node sinusoidal endothelium [16-18].

Previous investigations of common B cell markers (CD19, CD20, CD22, CD79b) according to expression level and pattern clearly distinguished normal B lymphocytes from all forms of $B$ cell malignant transformations $[6,7$, $19,20]$. The differences are slightly more subtle when it comes to distinguishing numerous forms of BCLPDs and producing a correct diagnosis for your patient.

Mc Carron et al. and Cabezudo et al. found that expression of CD79b is decreased in B-cell CLL compared with other BCLPDs, and proved to be a good discriminative marker between CLL and MCL as both CD5 positive BCLPDs $[2,20]$. Our findings of low/absent CD79b in CLL correlate with these results, while high expression of CD79b in SLL correlates with the results these authors described as typical for lymphoma cells. Jasper et al. and Huang et al. found that based upon CD22 ABC (antibody bound per cell) values, CD22 expression is lower in CLL, ALL, MCL, and FCL (in order from lowest to highest) than in normal B cells but is markedly increased in HCL. Our results show a difference between CLL and SLL cells as well, detecting low CD22 in CLL cells and medium CD22 in SLL cells. Expression of CD20 is higher in lymphomas [follicular lymphoma (FL), diffuse large B cell lymphoma (DBCL), mantle cell lymphoma (MCL) etc.], and lower in CLL than in normal $B$ lymphocytes [7, 12, 13, 20, 21]. Our results confirm the low expression rate in CLL cells, but the expression of CD20 in SLL cells correlates with other lymphomas.

From the diagnostic point of view, the quantitative estimation of CD79b together with that of CD5 seems to be discriminative between CLL and non-CLL cases whilst the antigen density of CD19 is a good discriminative marker between leukemic and normal B-cells [20]. CLL has a tendency to express dim CD20, dim slg, and absent or dim $\mathrm{CD} 79 \mathrm{~b}$ expression. These expression levels were shown to be statistically much lower in CLL than non-CLL BCLPDs, including MCL $[2,19,20,22]$, but according to our investigation they also show a difference with SLL as well. However, bright CD5, together with CD19 and dim slg, discriminate CLL and SLL from all other BCLPDs [19, 20].

From the other, functional point of view, low or absent CD79b, together with the low expression of CD22 and CD20, might explain the abnormal signal transduction of CLL cells similar to that of anergic B lymphocytes, as well as the low rate of apoptosis, which cannot be said for SLL cells $[8,11]$. Expression of CD22 also makes a significant biological difference between these cells, which may explain the tendency of SLL cells to populate lymph nodes [16-18]. Higher expression of CD79b and CD20 suggests that SLL cells are slightly more mature and more effective than CLL cells $[9,12]$.

This study shows that there is a difference in the expression level of common B cell markers on the CLL and SLL cells in peripheral blood. The high expression level detected on the SLL cells correlates with the expression level of common B cell markers on most lymphoma cells. According to the presented functional importance of these markers, CLL and SLL cells may exhibit different behavior. Though some papers suggest that there is no difference in outcome regardless of whether these patients were treated with recommended CLL or SLL chemotherapy protocols $[23,24]$, due to our results future therapy measures should be investigated for CLL and SLL separately.

The study was approved by the Ethical Committee of the Clinical Center Kragujevac. All patients gave their written informed consent according to the Declaration of Helsinki.

\section{Authors declare no conflict of interest.}

This study was supported by grant III41010 from the Ministry of Education, Science and Technological Development, Republic of Serbia. Also, our study was supported by grant JP 11/12 from the Medical Faculty, University of Kragujevac, Republic of Serbia.

\section{References}

1. Hallek M, Cheson BD, Catovsky D, et al. Guidelines for the diagnosis and treatment of chronic lymphocytic leukemia: a report from the International Workshop on Chronic Lymphocytic Leukemia updating the National Cancer Institute-Working Group 1996 guidelines. Blood 2008; 111: 5446-56.

2. McCarron KF, Hammel JP, Hsi ED. Usefulness of CD79b expression in the diagnosis of B-cell chronic lymphoproliferative disorders. Am J Clin Pathol 2000; 113: 805-13.

3. Moreau EJ, Matutes E, A'Hern RP, Morilla AM, Morilla RM, OwusuAnkomah KA, Seon BK, Catovsky D. Improvement of the chronic lymphocytic leukemia scoring system with the monoclonal antibody SN8 (CD79b). Am J Clin Pathol 1997; 108: 378-82.

4. Palma M, Kokhaei P, Lundin J, Choudhury A, Mellstedt H, Osterborg A. The biology and treatment of chronic lymphocytic leukemia. Ann Oncol 2006; 17: x144-x54.

5. Rawstron AC, Shingles J, de Tute R, Bennett F, Jack AS, Hillmen P. Chronic lymphocytic leukaemia (CLL) and CLL-type monoclonal B-cell lymphocytosis (MBL) show differential expression of molecules involved in lymphoid tissue homing. Cytometry B Clin Cytom 2010; 78B: S42-S6.

6. Jasper GA, Arun I, Venzon D, Kreitman RJ, Wayne AS, Yuan CM, Marti GE, Stetler-Stevenson M. Variables affecting the quantitation of CD22 in neoplastic B cells. Cytometry B Clin Cytom 2011; 80B: 83-90.

7. Ginaldi L, De Martinis M, Matutes E, Farahat N, Morilla R, Catovsky D. Levels of expression of CD19 and CD20 in chronic B cell leukaemias. J Clin Pathol 1998; 51: 364-9.

8. Matutes E, Polliack A. Morphological and immunophenotypic features of chronic lymphocytic leukemia. Rev Clin Exp Hematol 2000; 4: 22-47.

9. Dornan D, Bennett F, Chen Y, et al. Therapeutic potential of an anti-CD79b antibody-drug conjugate, anti-CD79b-vc-MMAE, for the treatment of non-Hodgkin lymphoma. Blood 2009; 114: 2721-9.

10. D’Arena G, Cascavilla N, Musto P, Colella Bisogno R, Pistolese G, Carotenuto M. CD79b expression in B-cell chronic lymphocytic leukemia. Haematologica 2000; 85: 556-7.

11. Alfarano A, Indraccolo S, Circosta P, et al. An alternatively spliced form of CD79b gene may account for altered B-cell receptor expression in B-chronic lymphocytic leukemia. Blood 1999; 93: 2327-35.

12. Almasri NM, Duque RE, Iturraspe J, Everett E, Braylan RC. Reduced expression of CD20 antigen as a characteristic marker for chronic lymphocytic leukemia. Am J Hematol 1992; 40: 259-63. 
13. Hsi ED, Kopecky KJ, Appelbaum FR, Boldt D, Frey T, Loftus M, Hussein MA. Prognostic significance of CD38 and CD20 expression as assessed by quantitative flow cytometry in chronic lymphocytic leukaemia. Br I Haematol 2003; 120: 1017-25.

14. Beers SA, Chan CH, French RR, Cragg MS, Glennie MJ. CD20 as a target for therapeutic type I and II monoclonal antibodies. Semin Hematol 2010; 47: 107-14.

15. Cesano A, Gayko U. CD22 as a target of passive immunotherapy. Semin Oncol 2003; 30: 253-7.

16. Moyron-Quiroz JE, Partida-Sánchez S, Donís-Hernández R, Sandoval-Montes C, Santos-Argumedo L. Expression and function of CD22, a B-cell restricted molecule. Scand I Immunol 2002; 55: 343-51.

17. Floyd H, Nitschke L, Crocker PR. A novel subset of murine B cells that expresses unmasked forms of CD22 is enriched in the bone marrow: implications for B-cell homing to the bone marrow. Immunology 2000; 101: 342-7.

18. Walker JA, Smith KG. CD22: an inhibitory enigma. Immunology 2008; 123: 314-25.

19. D’Arena G, Musto P, Cascavilla N, Dell'Olio M, Di Renzo N, Carotenuto M. Quantitative flow cytometry for the differential diagnosis of leukemic B-cell chronic lymphoproliferative disorders. Am J Hematol 2000; 64: 275-81.

20. Cabezudo E, Carrara P, Morilla R, Matutes E. Quantitative analysis of CD79b, CD5 and CD19 in mature B-cell lymphoproliferative disorders. Haematologica 1999; 84: 413-8.

21. Huang J, Fan G, Zhong Y, Gatter K, Braziel R, Gross G, Bakke A. Diagnostic usefulness of aberrant CD22 expression in differentiating neoplastic cells of B-cell chronic lymphoproliferative disorders from admixed benign $B$ cells in four-color multiparameter flow cytometry. Am J Clin Pathol 2005; 123: 826-32.

22. Olejniczak SH, Stewart CC, Donohue K, Czuczman MS. A quantitative exploration of surface antigen expression in common B-cell malignancies using flow cytometry. Immunol Invest 2006; 35: 93-114.

23. Tsimberidou AM, Wen S, O'Brien S, et al. Assessment of chronic lymphocytic leukemia and small lymphocytic lymphoma by absolute lymphocyte counts in 2,126 patients: 20 years of experience at the University of Texas M.D. Anderson Cancer Center. J Clin Oncol 2007; 25: 4648-56.

24. Asplund SL, McKenna RW, Howard MS, Kroft SH. Immunophenotype does not correlate with lymph node histology in chronic lymphocytic leukemia/small lymphocytic lymphoma. Am J Surg Pathol 2002; 26: 624-9.

\section{Address for correspondence}

Danijela Jovanovic, MD

Hematology Clinic

Clinical Centre Kragujevac

Zmaj Jovina 30

34000 Kragujevac, Serbia

e-mail: daziv81@yahoo.com

Submitted: 8.07 .2013

Accepted: 7.08 .2013 